\title{
Extracorporeal Circulation Membrane Oxygenation Therapy for Acute Respiratory Diseases
}

\author{
Javier Kattan Said, Álvaro González Morandé, \\ and Andrés Castillo Moya
}

\section{Contents}

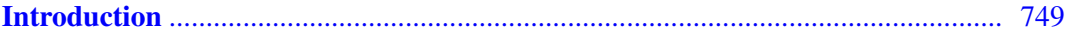

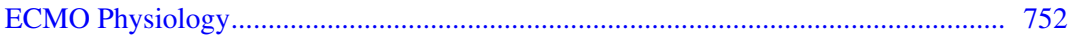

Selection Criteria for Applying ECMO ……………...................................... 753

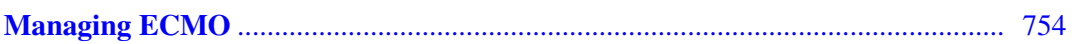

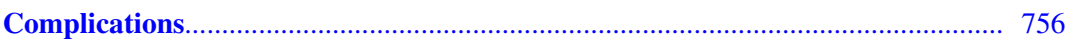

Prognosis and ECMO Programs in Latin America _.............................................. 756

Conclusions and Future Considerations......................................................... 758

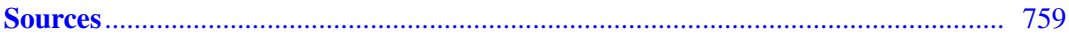

\section{Introduction}

Extracorporeal circulation membrane oxygenation (ECMO), or extracorporeal life support (ECLS), is a therapy that uses a modified partial cardiopulmonary bypass to give pulmonary or

J. Kattan Said · A. Castillo Moya $(\bowtie)$

Department of Pediatrics, School of Medicine, Pontificia Universidad Católica de Chile,

Santiago, Chile

e-mail: kattan@med.puc.cl; acastill@med.puc.cl

Á. González Morandé

Pediatrics, Neonatology, Pontificia Universidad

Católica de Chile, Santiago, Chile

e-mail: alvgonza@med.puc.cl cardiac support over an extended period of time, generally ranging from 1 to 4 weeks. It is used on patients with reversible pulmonary failure caused by lung, heart, and other diseases. ECMO 'makes time' for resting the lungs or heart, thus creating a chance for recovery. Since ECMO therapy is invasive, there are potential risks associated with it, which is why there are criteria to choose patients with a prediction of mortality between $50 \%$ and $100 \%$. The ideal candidate has a high value in their prediction of mortality but suffers from a potentially reversible lung or cardiovascular injury.

The first survivor of ECMO therapy was treated in 1971 by J. Donald Hill, who used a Bramson oxygenator on an adult patient with multiple injuries. This therapy was, however, abandoned because of its poor results. Years later, the therapy reemerged for newborn and 
pediatric patients due to Robert Bartlett's efforts, who in 1975, at Orange County Medical Center, treated his first newborn patient who survived this type of therapy, an abandoned newborn who suffered from respiratory distress syndrome. The use of this therapy on newborns increased during the late 1980s, with survival rates close to $80 \%$ in patients with a predicted mortality between $60 \%$ and $80 \%$. Because of this increase in usage on newborn patients, a voluntary alliance between active ECMO centers was born in 1989, giving form to the Extracorporeal Life Support Organization (ELSO). Added to the good results in newborns since the 1990s, there has been a considerable increase in pediatric patients treated with ECMO, with over 14,000 registered cases, claiming a survival rate of up to $66 \%$ in pediatric patients suffering from respiratory conditions.

By the year 2009, around $80 \%$ of patients treated with ECMO were newborns or children. During the past few years, ECMO on adult patients suffering from respiratory conditions increased progressively by $1000 \%$, and ECMO on pediatric patients suffering from respiratory conditions increased by $100 \%$, which is partially explained by the influenza H1N1 pandemic and the new evidence emerged from controlled studies in adults.

During the 1990s, new therapies against cardiorespiratory diseases were developed, such as high-frequency oscillatory ventilation (HFOV), surfactant, or inhaled nitric oxide (iNO). These therapies, in association with ECMO centers, have managed to significantly lower morbidity in more developed countries.

During the past decade, ECMO has been used as a rescue therapy in almost 800 newborns reported to ELSO every year. These newborns did not respond to intensive care with HFOV or iNO. Currently, ECMO use rate for newborns suffering from respiratory failure in the United States sits at around 1 per every 6000 living newborns This therapy has shown a clear increase in global survival rate (Figs. 73.1 and 73.2), better long-term quality of life, and being more costeffective for newborns with severe respiratory failure.
ECMO survival Discharge survival

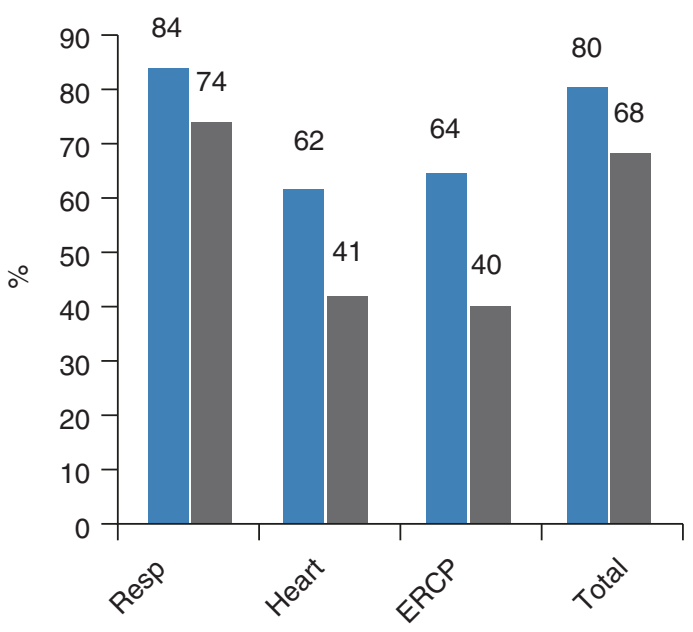

Fig. 73.1 Survival rate of newborns. Survival rate of 34,650 newborns after reported ECMO to the Extracorporeal Life Support Organization (ELSO), grouped by cause of admission to ECMO (ERCP Extracorporeal cardiopulmonary resuscitation)

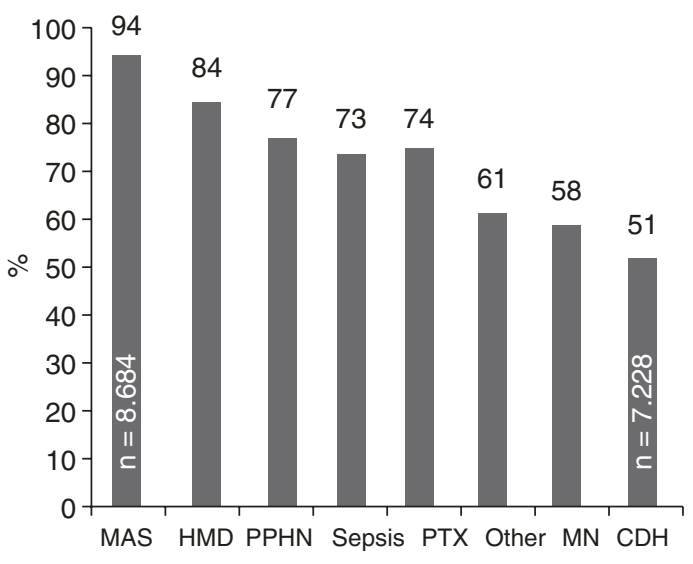

Fig. 73.2 Survival rate of Nnwborns. Survival rate to discharge of 27,728 newborns treated with ECMO, reported to ELSO by respiratory condition (MAS Meconium aspiration syndrome, $H M D$ Hyaline membrane disease, PPHN Persistent pulmonary hypertension of the newborn, $P T X$ Pneumothorax, $P N$ Pneumonia, $C D H$ Congenital diaphragmatic hernia)

For pediatric patients with respiratory conditions, the indications that lead to using ECMO are more diverse and harder to define than during the newborn stage, but during the past few years there has been an increase in the number of reported cases to ELSO, with close to 350 cases a 
year displaying a global survival rate of $57 \%$ at the discharge (Figs. 73.3 and 73.4).

It is worth noting that survival rate in pediatric patients varies according to the disease that determined the connection, with survival rates reported as high as $83 \%$ for severe asthma attack. Acute hypoxic respiratory failure is the most frequent

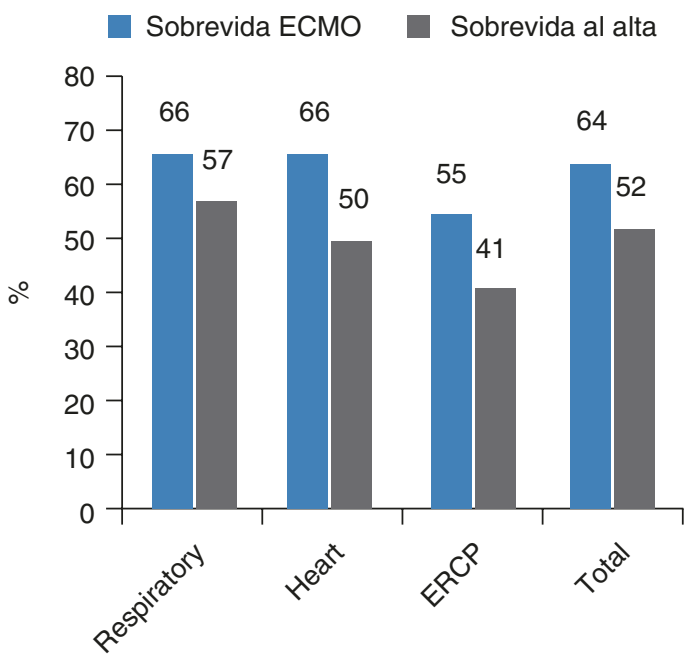

Fig. 73.3 Survival rate of pediatric patients. Survival rate of 16,253 pediatric patients after ECMO reported to the Extracorporeal Life Support Organization (ELSO), grouped by cause of admission to ECMO (ERCP Extracorporeal cardiopulmonary resuscitation) pathophysiological mechanism leading to respiratory ECMO, of which viral pneumonia takes first place $(22 \%)$, followed by respiratory failure $(18 \%)$, bacterial pneumonia (10\%), acute respiratory distress, and aspiration pneumonia. Acute viral pneumonia shows a survival rate of up to $70 \%$ as described for VRS, with an average of $64 \%$ for viral pneumonia. Survival rates are also high in the pediatric group for aspiration pneumonia and post-traumatic ARDS (Fig. 73.5). Patients are oftentimes admitted because of immunosuppression and suspected sepsis. These patients usually display multi-organ failure. Pediatric patients with the worst prognosis are those who have received bone marrow transplants, those who have suffered from Bordetella pertussis induced pneumonia and pulmonary hypertension, and those admitted to ECMO with multi-organ failure, as opposed to the positive prognosis for those who only show isolated lung involvement.

In 1972, Bartlett reported the first case of successful prolonged post-operative cardiac support in a 2-year-old patient who suffered from postsurgery heart failure after a Mustard procedure because of transposition of great arteries. At present, more than half of patients requiring perioperative heart ECMO are those suffering from complex cyanotic congenital heart diseases. The largest group of patients requiring ECMO sup-
Fig. 73.4 Survival rate of pediatric patients. Survival rate of 6569 discharged pediatric patients treated with ECMO reported to ELSO by respiratory condition (ARDS Acute respiratory distress syndrome)

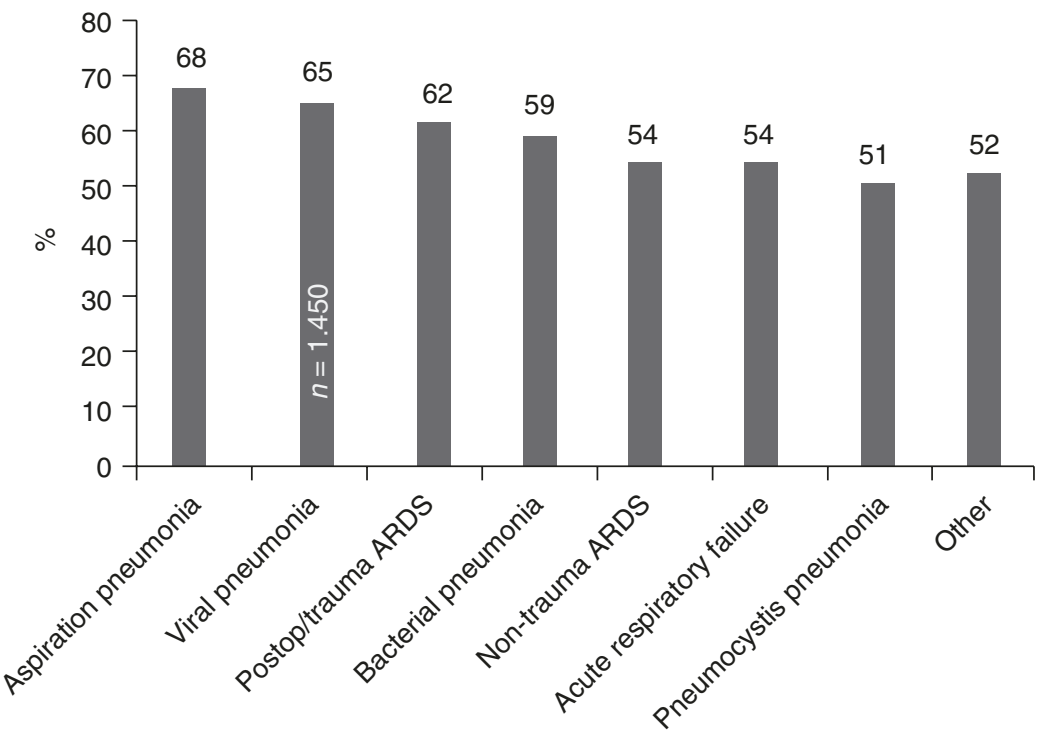




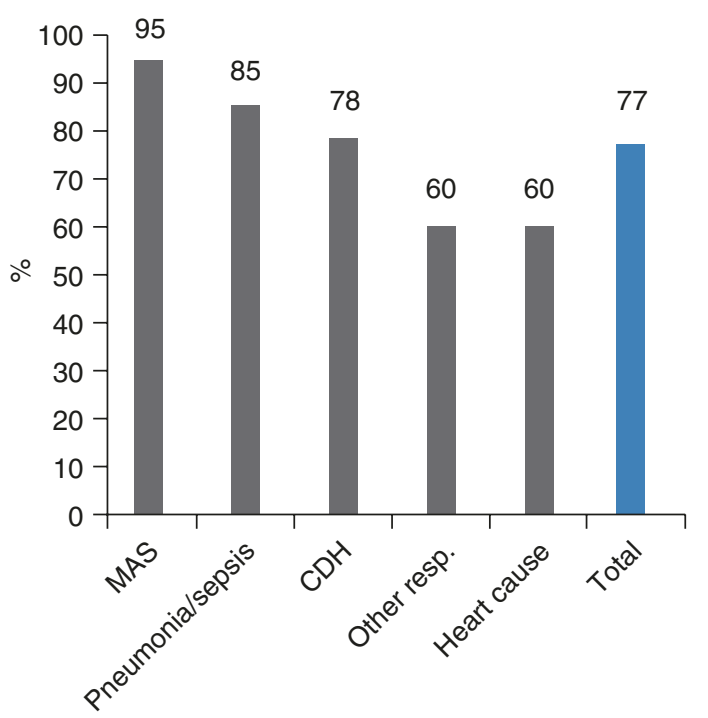

Fig. 73.5 Survival rate to discharge. Survival rate to discharge of 143 patients (123 newborns and 20 pediatric patients) treated in the Newborn-Pediatric ECMO Program at the Clinical Hospital of the Pontificia Universidad Católica de Chile (ECMO-UC) 2003-2014, reported to ELSO by main diagnosis (MAS Meconium aspiration syndrome; $C D H$ Congenital diaphragmatic hernia)

port is those who, after cardiotomy, present complete AV canal (20\%), complex single ventricle anomaly (17\%), and tetralogy of Fallot (14\%). Among the chief causes requiring perioperatory heart ECMO are hypoxia (36\%), cardiac arrest (24\%), and failure after leaving extracorporeal circulation support (14\%).

\section{ECMO Physiology}

During extracorporeal circulation support, blood is drained from the patient to an outside pump (either a roller or a centrifugal pump), which is pushed through an exchange membrane (a silicone or a polymethylpentene oxygenator) for oxygenation and $\mathrm{CO}_{2}$ removal. Then it passes through a heat exchanger and finally returns the blood to the patient's blood flow (Fig. 73.6). This therapy requires anticoagulation of the circuit and the patient through heparin administered to the ECMO circuit so as to avoid the activation of the coagulation cascade in the system. In addition to that, a variety of pressure, flow, bubble, and temperature monitors are used during therapy. It is of vital importance to continually monitor coagulation through the hourly measurement of activated clotting time (ACT) and the measurement of anti-factor Xa, fibrinogen, platelet count, PT, APTT and, in some patients, anti-thrombin III levels, and a thromboelastography.

There are in principle two different forms of ECMO:

(a) Veno-arterial (VA): Blood is drained from the right atrium through a cannula inserted into the right internal jugular vein, the femoral vein, or directly to the right atrium; and it is picked back up at the thoracic aorta through a right carotid cannula, a femoral cannula or an aortic cannula. VA ECMO provides heartlung support. It is common to use a transthoracic cannula (right ventricle and aortic cannula) in patients who have endured heart operations.

(b) Veno-venous $(V V)$ : Blood is drained from the right atrium through the posterior and inferior orifices of a double-lumen cannula inserted into the right jugular vein and returned to the same right ventricle through the anterior orifices of the cannula, which are pointed toward the tricuspid valve. One of the limits of this method lies in the recirculation of already oxygenized blood through the double lumen cannula. This has been corrected with the new design for $\mathrm{VV}$ cannulas. VV ECMO may also be performed in older children through the use of two cannulas, draining blood from the jugular vein and returning it through the femoral vein. VV ECMO requires a well-functioning heart. This modality of ECMO prevents cannulation in the carotid or femoral arteries, thus reducing complications arising from cannulation or ligation of these arteries, as well as those arising from air entering the ECMO circuit. This method has seen an increase in usage during recent years, covering $40 \%$ and $50 \%$ of respiratory cases in newborn and pediatric patients, respectively. Oxygen is delivered during ECMO by the combination of blood oxygenation through the membrane, 


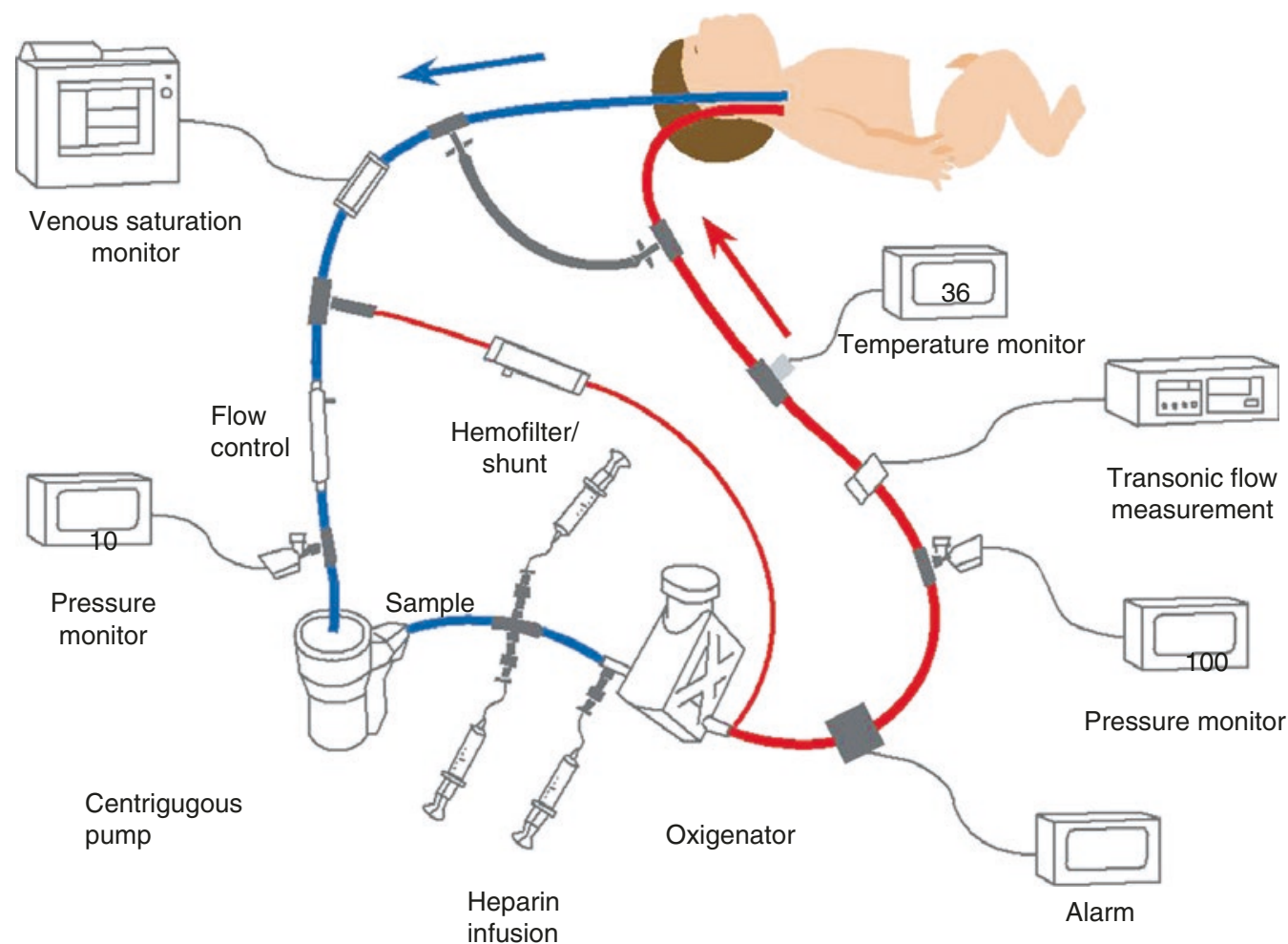

Bubble detector

Fig. 73.6 Diagram of veno-arterial ECMO with pump and oxygenator. Venous blood is obtained from the right atrium through the right internal jugular vein. It is then pumped, oxygenized, heated and returned to the aorta

blood flowing through the extracorporeal circuit, native lung oxygenation, and native heart output. In turn, oxygenation at the ECMO membrane is a function of its geometry, material composition and thickness, blood and $\mathrm{FiO}_{2}$ laminar thickness, time of permanence of red blood cells in the exchange area, hemoglobin concentration, and $\mathrm{O}_{2}$ saturation. On the other hand, $\mathrm{CO}_{2}$ removal during ECMO is a function of the geometry, materials, and surface area of the membrane, blood $\mathrm{PCO}_{2}$ and, to a lesser extent, it depends on blood and gas flows through the membrane.

- In a VA ECMO, the bypass generates an essentially non-pulsatile blood flow. In this way, as the blood flow to the extracorporeal circuit increases, the pulse wave decreases, completely ceasing when it reaches a $100 \%$ through the right carotid artery. (Diagram used with permission from ECMO Manual of the Children's National Medical Center, George Washington University, Washington DC, 2010)

bypass, except for occasional waves. However, it is normal for VA ECMO to only involve about an $80 \%$ bypass, allowing a blood flow of $20 \%$ or more through the left heart and lungs, resulting in a reduced but visible pulse wave. The kidney is without doubt the most affected organ by the absence of pulsatility, producing an antidiuretic effect because of juxtaglomerular stimulation. In addition, non-pulsatile flow has been linked to stimulation of the pressure receptors in the carotid sinus, causing a large release of catecholamines, with damaging effects to microcirculation.

\section{Selection Criteria for Applying ECMO}

Selection criteria differ for newborn (Table 73.1) or pediatric patients (Table 73.2), depending on 
Table 73.1 Selection criteria for newborns

Gestational age $\geq 34$ weeks

Weight at birth $\geq 2000$ grams

Unresponsive to maximum medical care (including

HFOV, iNO, surfactant)

Reversible cardiopulmonary condition

Mechanical ventilation $\leq 14$ days

High pulmonary mortality (50-100\%), considering:

Oxygenation index $(\mathrm{OI})>35-40$ for $4-6$ hours (iNO, HFOV)

$\mathrm{PaO}_{2}<40 \mathrm{mmHg}$ for $4 \mathrm{~h}\left(100 \% \mathrm{O}_{2}\right)$

$\mathrm{OI} \geq 25$ after $72 \mathrm{~h}$ with HFOV-iNO

Unmanageable metabolic acidosis ( $\mathrm{ph}<7.15$ for $2 \mathrm{~h}$ )

Reduced cardiac output with reversible etiology

Impossibility to wean from cardiopulmonary bypass

As a bridge for heart transplant

No untreatable congenital cardiopathy or injuries after heart surgery

Absence of major intracranial hemorrhage ( $\geq$ III degree)

Absence of uncontrollable hemorrhage

No evidence of irreversible brain damage

No malformations or genetic syndromes with fatal prognosis

Table 73.2 Selection criteria for pediatric patients

$\mathrm{OI}>40$ for $4-6 \mathrm{~h}$ or $\mathrm{PaO}_{2} / \mathrm{FiO}_{2}<60$ during mechanical ventilation (MCV or HFOV)

OI $>35$ for $>12 \mathrm{~h}$. High settings for mechanical

ventilation, considering:

Pressure plateau $>35 \mathrm{~cm} \mathrm{H}_{2} \mathrm{O}$ for 8-12 hours

PEEP $>15$

Average airway pressure $>20-25 \mathrm{~cm} \mathrm{H}_{2} \mathrm{O}$ in MCV or $>30-35 \mathrm{~cm} \mathrm{H}_{2} \mathrm{O}$ in HFOV

Hypercapnic respiratory failure with $\mathrm{pH}<7.1$ for

4-6 hours despite maximum medical care

Acute deterioration with optimal therapy

whether the primary cause of admission is cardiac or respiratory. These are general criteria and must be individualized for each patient, assessing the risks and benefits of applying ECMO. The basic selection criteria for pediatric patients with respiratory failure are similar to those for newborns, with particular emphasis on whether the patient faces a serious pulmonary condition with a high risk of death, or whether it is a process that can be reversed through respiratory, gasometrical, and hemodynamic rest. On the other hand, there are general exclusion criteria (Table 73.3), though none of them are absolute and must be
Table 73.3 General exclusion criteria in the pediatric population

Over 14 days with mechanical ventilation

Over 7 days of high ventilatory settings, given the possibility of iatrogenic lung damage

Acute or irreversible brain damage

Chronic acute or irreversible lung disease

Severe coagulopathy or uncontrolled bleeding

Untreatable congenital cardiopathy

Chromosomal syndrome with limited prognosis

Cardiopulmonary arrest unless there is an extracorporeal cardiopulmonary resuscitation program (post-arrest ECMO or E-RCP) at the ECMO center

discussed by the ECMO team. In summary, selection criteria have evolved and continue to do so as a result of discussion, debate, experience, and the emergence of new treatments and techniques. Currently, there are no unique or exclusive criteria, and not withstanding general inclusion criteria, the decision to exclude a patient must be discussed by the team, taking into account all possible points of view, without excluding any party from this discussion.

\section{Managing ECMO}

Before a patient is admitted to ECMO, both indications and exclusion criteria must always be assessed, making it essential to perform a patient evaluation prior to ECMO, including at least age and anthropometry, neurological status (general exam, pupillary reactivity, electroencephalogram, history of seizures, head ultrasound, and ideally a brain CT scan for pediatric patients); respiratory and cardiovascular evaluation (oxygenation index, vasoactive support and, if possible, an echocardiography), electrolytic and acid-base evaluation, coagulation and renal function evaluation, evaluation of infectious parameters, and an assessment of current or previous vascular accesses.

The initial ECMO parameters seek to achieve a bypass of at least $50 \%$ or more of estimated cardiac output (with cardiac output estimated at $200 \mathrm{~mL} / \mathrm{kg} / \mathrm{min}$ for newborns, $150 \mathrm{~mL} / \mathrm{kg} / \mathrm{min}$ for pediatric patients under $10 \mathrm{~kg}$ and $2400 \mathrm{~mL} /$ $\mathrm{m} 2 \mathrm{sc} / \mathrm{min}$ for patients over $10 \mathrm{~kg}$ ), and are 
adjusted to maintain adequate oxygenation, arterial pressure, and acid-base state. In patients with heart failure, VA-ECMO is the preferred choice. At present, when proper cardiac function is preserved and the main pathology is pulmonary, VV-ECMO is considered to be of assistance in oxygenation and ventilation.

The main points of access will depend on the modality of ECMO being used. For VA-ECMO, the right internal jugular vein and the right common carotid artery may be used as well as the femoral veins and arteries. For VV-ECMO, either the right internal jugular vein and the femoral vein may be used, or a long unique double-lumen cannula inserted into the right internal jugular vein, reaching the right atrium. In post-operative cardiac patients, direct transthoracic cannulation is common.

At present, most oxygenators use polymethylpentene fibers. These have microscopic pores, making the exchange of gases easier and avoiding 'dripping'. They are quickly and easily primed, displaying low trans-membrane pressure. This avoids the loss of proteins and other components. In fact, by having a more homogenous surface they produce less hemolysis and preserve platelet activity.

The strength with which blood is pumped through the circuit depends on the pumps, which may be of the roller or centrifugal type. Centrifugal pumps are increasingly preferred given the advantage of not being occlusive, which causes less pressure on the circuit and less hemolysis. They do not need a reservoir, are primed faster, and help preserve the circuit for a longer period. Their man disadvantage lies in their high cost, which is why some units still use roller pumps.

ECMO treatment may vary between 1 and 3 weeks or even longer. In January 2014, pediatric respiratory ECMO lasted an average of 11.6 days, with a described maximum of 129 days. On the other hand, pediatric cardiac ECMO generally take less time, with an average of 6.7 days with a described maximum of 120 days. For respiratory patients, improvement is generally noticed after a few days or even weeks.
Meticulous attention to all aspects of the patient is essential. Frequent checks of blood gas are required for the patient and the ECMO circuit, as well as clotting and kidney function checks, and an ultrasound assessment of the brain to identify intracranial hemorrhage and cerebral infarction.

Both arterial and venal saturation of the patient must be continually monitored, taking into account saturation levels of the blood extracted from the patient before it enters the oxygenator (ideally over 70-75\%). Saturation of the superior vena cava should also be monitored. However, something to keep in mind for these last two is that there should be no doubt that there is no unnoticed source of short circuit of oxygenated blood.

Anticoagulation is essential in managing patients, both for ensuring an adequate duration of the circuit and to avoid severe bleeding from excessive anticoagulation. In this regard, hourly managing of levels of ACT from 180 to $200 \mathrm{sec}$., with a platelet count of over 100,000 and fibrinogen levels of over $150 \mathrm{mg} / \mathrm{dl}$ is thought to be adequate.

At least during the first days, patients must receive adequate sedoanalgesia with continuous opiate infusions and benzodiazepines, considering the eventual use of muscle relaxants in some cases. There has been a current increase in considering the benefits of using a smaller amount of anesthesia, with some groups even keeping patients awake during the procedure. This particularly applies to older patients.

Once ECMO support is being used, the mechanic ventilator settings should be set to rest. Despite the lack of a proper definition for this, it is considered so for pediatric patients with PEEP of 6-8 in order to avoid unrecruited lung, tidal volumes that do not surpass $8 \mathrm{~mL} / \mathrm{kg}$, and an FR of $12-18$ per minute with FiO2, usually at $21 \%$. In addition, emergency settings must always be kept in mind in case of an ECMO malfunction, in which case $\mathrm{FiO} 2$ must be considered at $100 \%$.

During ECMO, water balance and diuresis are needed as soon as possible, as well as volume overload prior to its commencement. Positive water balance and oligo-anuria must be avoided, 
because once hemodynamics is stable, water balance should become negative by using a proportional diuresis, and afterwards, neutral. If these objectives are not achieved, or if the patient develops an oligo-anuric kidney injury, continuous hemofiltration or hemodiafiltration should be considered for as long as diuretics are used and if adequate hemodynamics did not manage to restore an adequate kidney function to achieve the required balance.

Finally, prophylactic antibiotics are not generally considered for this treatment. Using antibiotics in most patients depends either on the underlying disease, which for a large percentage of patients corresponds to an infectious disease, or if there is a suspicion of superimposed infections and as the result of surveillance or secondary cultures under clinical suspicion of infection.

As the patient's condition improves, ECMO support is gradually reduced. The patient is decannulated when tolerance to minimal ECMO support is achieved ( $10 \%$ bypass in VA-ECMO) with low to moderate mechanical ventilation parameters. ECMO treatment generally lasts between 5 and 10 days for newborn patients with respiratory diseases, though this is extended in cases of congenial diaphragmatic hernia, bacterial pneumonia, and myocarditis (10-11 days in average).

\section{Complications}

The ECMO procedure presents varying complication risks as a consequence of the severity of the condition of the patient, the use of anticoagulants, and changes to the blood flow (lower pulsatility blood flow). Among the most common complications are hemorrhage (surgical site $6 \%$, pulmonary $4 \%$, gastrointestinal $2 \%$ ), infarction or brain hemorrhage (9\% and 5\% respectively), convulsions $(11 \%)$, cardiac dysfunction (myocardial stunning 6\%, arrhythmia 4\%), kidney failure (4\%), sepsis (6\%), hyperbilirubinemia (9\%), arterial hypertension (12\%), and hemolysis (13\%). The most common complication with cardiac-based ECMO is, by far, the need for vasoactive drugs during extracorporeal support, followed by surgical site bleeding.
Intracranial hemorrhage is the primary cause of death during ECMO, and the appearance of convulsions is a sign of a poor prognosis. Additionally, there are complications arising from circuit failure either in the oxygenator or in the equipment used during ECMO.

\section{Prognosis and ECMO Programs in Latin America}

Post-ECMO survival in newborn patients varies depending on their underlying disease, though respiratory causes present the most positive outcome with a survival rate of close to $75 \%$ after discharge. ECMO treatment for meconium aspiration syndrome is usually of the veno-venous type, which is associated with a lower risk rate and fewer complications, such as cerebral infarction and convulsions, and to minor changes in blood flow patterns. During the past few years, ECMO has been used as a tool for cardiopulmonary resuscitation after cardiac arrest yielding mixed results, with a survival rate of close to $40 \%$.

Newborns were the first age group for which ECMO therapy showed vast superiority over maximum conventional therapy, as shown by a controlled and randomized multicenter study with 185 newborns suffering from severe respiratory insufficiency in 55 hospitals in the United Kingdom. This study showed that mortality and severe disability assessed after 1, 4, and 7 years of life decreased significantly after ECMO therapy (59\% for the conventional therapy group vs. $37 \%$ for the ECMO group). At the seventh year of follow-up, $76 \%$ of children presented normal cognitive development.

The most recent systematic reviews show that ECMO therapy used in close to term newborns with severe but potentially reversible respiratory failure significantly improves survival without increasing severe disability while also being costeffective when compared to other intensive care therapies. Regarding ECMO as a rescue therapy for congenital diaphragmatic hernia with severe respiratory failure, evidence from controlled prospective studies only suggests a decrease in early mortality. However, a meta-analysis of retrospec- 
tive studies and our own reported experience show a higher short- and long-term survival rate for congenital diaphragmatic hernia in units where ECMO is available.

Survival rate and neurological prognostic after 5 years in patients who underwent ECMO for noncardiac causes is generally very positive, but it worsens for lower gestational age groups, lower weight at birth, and higher oxygenation index (OI) before ECMO. Patients with a septic shock diagnosis and congenital diaphragmatic hernia display the worst survival rate and neurological evolution outcomes. However, it is the pre-existent factors and the severity of the condition of the newborn when entering ECMO that apparently determines the long-term neurological prognosis.

Long-term respiratory prognosis depends on the base etiology, degree of barotrauma, and length of exposure to oxygen. Between $10 \%$ and $30 \%$ of patients suffering from congenital diaphragmatic hernia have episodes of wheezing by age 10, with close to 50 suffering from hyper insufflation and episodes of airway obstruction. Pediatric post-ECMO survival rate is lower than that of newborns, but the respiratory failure group presents a better prognosis, particularly for patients with aspiration pneumonia, viral pneumonia, and post-operative ARDS or ARDS developed after traumatic injury. Viral pneumonia is the most common condition leading to pediatric ECMO, and among its etiologies, respiratory syncytial virus presents the highest post-ECMO survival rate at $70 \%$. On the other hand, patients suffering from pneumonia caused by other viruses and by Bordetella pertussis report lower survival rates of $56 \%$ and $39 \%$, respectively.

Given the evidence that shows an increased survival rate and the cost-effectiveness relation of this therapy, the newborn intensive care unit at the Pontifical Catholic University established a newborn-pediatric ECMO program following the standards advocated by the Extracorporeal Life Support Organization (ELSO) for patients with severe but reversible cardiovascular or respiratory insufficiency refractory to maximum conventional treatment. Work began in 1999 with the formation of a multidisciplinary team (composed of neonatologists, intensive care pediatricians, cardiac and pediatric surgeons, nurses, perfusionists, respiratory therapists, and psychologists) that had undergone training in ECMO centers affiliated with ELSO in the United States. At the same time, ECMO equipment was procured in order to establish the first ECMO units according to ELSO recommendations. The main selection criteria applied were: reversibility of lung or heart condition, failure to respond to maximum conventional treatment, weight ${ }^{3}$ of $2 \mathrm{~kg}$, gestational age of 34 weeks, oxygenation index $>40$, mechanical ventilation $<14$ days, and absence of severe brain injury or multi-organ failure. This is the first ECMO program in Latin America to join ELSO. From May 2003 to December 2014, the program treated 143 patients for both severe respiratory and cardiac pathologies. Of these patients, 77\% survived till hospital discharge and are currently being monitored. The 35 patients who died had as base diseases: $\mathrm{CDH}(n=13)$, congenital heart disease operated with failure to wean from cardiopulmonary bypass or arrhythmias $(n=9)$, persistent pulmonary hypertension secondary to sepsis, pneumonia, meconium aspiration syndrome, SP-B or ABCA3 deficiency or without a defined cause $(n=12)$, and pneumonia due to Bordetella pertussis $(n=1)$. Among patients treated with ECMO, a relevant group of children treated with $\mathrm{CDH}$ stands out with a survival rate of $78 \%$ (45/48) at discharge.

In order to understand the impact of establishing a neonatal ECMO program on the survival rate of newborns with severe respiratory failure in a developing country such as Chile, we studied the data of newborns with severe respiratory failure and OI $>25$ before and after ECMO treatment was available. ECMO was initiated for a newborn with acute refractory respiratory failure who did not respond to iNO or HVOF. Data compiled from treatment of 259 newborns were analyzed, resulting in a significant increase in survival rate, from $72 \%$ before ECMO to $89 \%$ during the ECMO period. During the ECMO period, 98 out of 159 patients with respiratory failure $(62 \%)$ were rescued using iNO or HFOV, while 61 (38\%) did not improve their condition; 52 out of these 61 patients underwent ECMO treatment. The survival rate at discharge after 
ECMO was $85 \%$. Severity of OI, late arrival to the referral center, presence of a pneumothorax, and a diagnosed $\mathrm{CDH}$ were associated with the need for ECMO treatment or death.

One hundred percent of the survivors from our program are currently undergoing a special ECMO follow-up program. Among neurological follow-up exams, Bayley II tests at 12-18 months show that over $90 \%$ of tested children had normal or slightly altered mental development indices (MDI), and over $70 \%$ of them had normal or slightly altered psychomotor development indices (PDI). In addition, no patient displayed disabling visual or auditive alterations

Regarding respiratory follow-up, $83 \%$ of patients had a normal or slightly altered clinical broncho-pulmonary evaluation at 12-18 months. During the first year of life, $30-40 \%$ of them required hospitalization because of acute respiratory syndrome. Close to one third of patients present bronchial hyperreactivity diagnosed through a methacholine test in the medium-term.

As happened in Chile, new neonatal-pediatric ECMO programs have been constituted during the past few years in high-complexity, highvolume centers in several countries in Latin America, such as Argentina, Colombia, Brazil, and Mexico, most of which have progressively joined ELSO, forming the Latin American chapter of ELSO in 2012. The mission of this new ELSO chapter is to contribute to the dissemination of ECMO therapy according to ELSO standards, practical and theoretical education through courses and workshops, as well as encouraging collaborative work between Latin American centers, of which 15 can be found across five different countries, with close to 200 newborns and 120 children as reported to ELSO by December 2014.

\section{Conclusions and Future Considerations}

ECMO therapy, or more broadly, extracorporeal life support (ECLS), is a standard therapy in neonatology and pediatrics with proven benefits in the short and long term. It can be incorporated to intensive therapy with positive results in develop- ing countries, but it must be implemented by high-complexity neonatal and pediatric centers with trained personnel and a high level of commitment.

Future patients to be treated with ECLS will progressively present more complex conditions, which is why new ECLS modalities will be required. These will have to be simpler, automatic, and with a lower need for anticoagulants so as to minimize risks and make its extended use possible. In that way newborns and children with severe conditions can be submitted to ECLS while waiting for heart or lung transplant, or as a bridge for ventricular assistance devices. Even premature newborns with severe cardiopulmonary failure can benefit from partial umbilical ECLS in the future. New low-resistance micropore oxygenators will not require the use of a pump, with the umbilical artery and vein used as an arteriovenous shunt. Moreover, newborns with $\mathrm{CDH}$ could go through an early ECLS admission in order to minimize lung damage and promote lung growth, using, for instance, growth factors or liquid ventilation with perfluorocarbon associated with ECLS. Some centers, such as Boston Children's Hospital, have applied ex-utero intrapartum treatment (EXIT) to ECMO for patients either with $\mathrm{CDH}$ and prenatal markers of poor prognosis, or in order to ensure an effective ventilation of newborns who do not possess a safe airway or for whom it is expected suffer from severe respiratory failure at birth $(\mathrm{CDH}$, cervical teratoma, airway pathologies, large pulmonary masses, bronchial cysts, etcetera).

The progressive increase of pediatric patients who benefit from the use of ECLS is worth noting, and it is something that has been increasingly determined by the growing number of pathologies that may indicate severe respiratory failure within this population. This has lead patients with sepsis, immunocompromised patients, patients suffering from heart failure and other complex pathologies, to be ECLS candidates. Continuous technical improvements have created a technique that is both safer and easier to perform, so it is expected that in the coming years we will witness a sustained increase in pediatric patients who can benefit from ECLS therapy. 
In this way, we hope that ECLS will enable us to keep assisting pulmonary or cardiac functioning in a more rational way while severe but reversible cardiopulmonary processes are repaired.

\section{Sources}

Alvarado-Socarras JL, Gomez C, Gomez A, Cruz M, Diaz-Silva GA, Nino MA. Current state of neonatal extra-corporeal membrane oxygenation in Colombia: de-scription of the first cases. Arch Cardiol Mex. 2014;84:121-7.

Arens J, Schoberer M, Lohr A, Orlikowsky T, Seehase M, Jellema RK, et al. NeonatOx: a pumpless extracorporeal lung support for premature neonates. Artif Organs. 2011;35:997-1001.

Bartlett R. Physiology of ECLS. In: Annich GMLW, MacLaren G, Wilson JM, Bartlett RH, editors. ECMO: extracorporeal cardiopulmonar support in critical care. 4th ed. Ann Arbor, Michigan: ELSO; 2012. p. 11-31.

Bohn D. Acute hypoxic respiratory failure in children. In: Annich GMLW, MacLaren G, Wilson JM, Bartlett $\mathrm{RH}$, editors. ECMO: extracorporeal cardiopulmonary sup-port in critical care. 4th ed. Ann Arbor, Michigan: ELSO; 2012. p. 41-72.

Dalton H, Butt W. Extracorporeal life support: An update of Rogers'textbook of pediatric intensive care. Pediatr Crit Care Med. 2012;13:461-71.

ELSO Data Registry. University of Michigan: Ann Arbor M. ECMO registry of the Extracorporeal Life Support Organization (ELSO). Ann Arbor Michigan: University of Michigan; 2015.

Fortenberry JD. The history and development of extracorporeal support. ECMO: extracorporeal cardiopulmonar support in critical care. 4th ed. Ann Arbor Michigan: ELSO; 2012. p. 1-10.

Hamutcu R, Nield TA, Garg M, Keens TG, Platzker AC. Long-term pulmonary sequelae in children who were treated with extracorporeal membrane oxygenation for neonatal respiratory failure. Pediatrics. 2004;114:1292-6.

Kattan J, Godoy L, Zavala A, Faunes M, Becker P, Estay A, et al. Improvement of survival in infants with congenital diaphragmatic hernia in recent years: effect of ECMO availability and associated factors. Pediatr Surg Int. 2010;26:671-6.
Kattan J, Gonzalez A, Becker P, Faunes M, Estay A, Toso $\mathrm{P}$, et al. Survival of newborn infants with severe respiratory failure before and after establishing an extracorporeal membrane oxygenation program. Pediatr Crit Care Med. 2013;14:876-83.

Keller RL, Steinhorn RH. Extracorporeal membrane oxygenation in resource-limited countries. Pediatr Crit Care Med. 2013;14:900-2.

McNally H, Bennett CC, Elbourne D, Field DJ. United Kingdom collaborative randomized trial of neonatal extracorporeal membrane oxygenation: follow-up to age 7 years. Pediatrics. 2006;117:e845-54.

Moreno GE, Magliola R, Pilan ML, Althabe M, Balestrini M, Lenz AM, et al. Mechanical circulatory support in pediatrics. Experience at the hospital de Pediatria Dr. Juan P. Garrahan. Argentina. Arch Cardiol Mex. 2014;84(4):256-61.

Morini F, Goldman A, Pierro A. Extracorporeal membrane oxygenation in infants with congenital diaphragmatic hernia: a systematic review of the evidence. Eur J Pediatr Surg. 2006;16:385-91.

Mugford M, Elbourne D, Field D. Extracorporeal membrane oxygenation for severe respiratory failure in newborn infants. Cochrane Database Syst Rev 2008: CD001340.

Paden ML, Rycus PT, Thiagarajan RR. Update and outcomes in extracorporeal life support. Semin Perinatol. 2014;38:65-70.

Pawlik TD, Porta NF, Steinhorn RH, Ogata E, de Regnier RA. Medical and financial impact of a neonatal extracor-poreal membrane oxygenation referral center in the nitric oxide era. Pediatrics. 2009;123:e17-24.

Peek GJ, Mugford M, Tiruvoipati R, Wilson A, Allen E, Thalanany MM, et al. Efficacy and economic assessment of conventional ventilatory support versus extracorpor-eal membrane oxygenation for severe adult respiratory failure (CESAR): a multicentre randomised controlled trial. Lancet. 2009;374:1351-63.

Smalley N, MacLaren G, Best D, Paul E, Butt W. Outcomes in children with refractory pneumonia supported with extracorporeal membrane oxygenation. Intensive Care Med. 2012;38:1001-7.

Thiagarajan RY, Bratton V. SL. Cardiac failure: principles and physiology. In: Annich GMLW, MacLaren G, Wilson JM, Bartlett RH, editors. ECMO: extracorporeal cardio-pulmonar support in critical care. 4th ed. Ann Arbor, Michigan: ELSO; 2012. p. 33-9.

Zabrocki LA, Brogan TV, Statler KD, Poss WB, Rollins MD, Bratton SL. Extracorporeal membrane oxygenation for pediatric respiratory failure: survival and predictors of mortality. Crit Care Med. 2011;39:364-70. 